\title{
A Theory of Our Universe
}

\author{
Robert M. L. Baker Jr. \\ Transportation Sciences Corporation, Pam Desert, California, USA \\ Email: DrRobertBaker@GravWave.com
}

How to cite this paper: Baker Jr., R.M.L. (2020) A Theory of Our Universe. Journal of High Energy Physics, Gravitation and Cosmology, 6, 609-622. https://doi.org/10.4236/jhepgc.2020.64041

Received: July 21, 2020

Accepted: August 14, 2020

Published: August 17, 2020

Copyright (C) 2020 by author(s) and Scientific Research Publishing Inc. This work is licensed under the Creative Commons Attribution International License (CC BY 4.0).

http://creativecommons.org/licenses/by/4.0/

\begin{abstract}
Contemporary theories of our Universe, such as the Friedmann-LemaitreRobertson-Walker (FLRW) model of the cosmos, assume that time marches on at a uniform, constant pace from its very beginning. But what if that is not the case? It is proposed that our Universe is not a "Big Bang", but rather a "Big Rollout" in space and time, spacetime, from the shortest meaningful length, Planck Length, and the shortest meaningful measure of time, Planck Time. It is speculated that time and dimensions, spacetime, grow in concert very rapidly at first. The fundamental equation, which relates the change in the space dimensions to the change in the speed of time at the beginning of time for the new Theory, is derived. Spacetime rolls out initially at light speed. As time increases, the rate of change of the speed of time could be erratic, that is although in general, it slows (rate of time slows approaching zero at the end of time), its rate of change could decelerate, pause or perhaps accelerate for a while, no need however, for dark matter or dark energy.
\end{abstract}

\section{Keywords}

Cosmology Theory, Early Universe, Dark Matter, High-Frequency Relic Gravitational Waves

\section{Introduction}

In this paper a Theory of our Universe is presented in an unusual manner since this new theory is best understood by pictures rather than by a large number of equations. First of all, it is important to understand the concept of time that is employed in the theory. Not only is time taken to be a dimension of spacetime, but it has a literal meaning as measured by looking at a stopwatch or a wristwatch-a "measuring device" that can appear, like some clocks, to run too fast or too slow. In fact, time is considered in this approach to be similar to the frame-rate of a movie. One can photograph a movie with the frame rate going fast, regular rate or at a slow frame rate. As an example, consider a movie taken 
in the past of a weight-lifting contest, for example taken billions of years ago. In every section or sequence in the movie the contestants move the weights in accordance with the laws of Physics, yet when the film is viewed from a film taken from a variable-frame-rate camera, the athlete's movements do not always match the reality that the movie viewer expects to see! Secondly, if we observed a variable-rate camera movie taken in the past of a Physics Laboratory, then we might view an experiment to measure the acceleration of gravity appear to obtain incorrect results and likewise an experiment to measure the speed of light could yield inconsistent values. Of course when all the experiments are photographed by a camera run at the usual frame rate, they yield the expected usual results. We could take a variable-frame-rate movie of a more sophisticated laboratory that measures general-relativity effects including those associated with "World Lines", "Proper Time," etc. Every film clip sequence or even individual frame sequences could be different: first a fast frame rate, then a slow frame rate, then a fast frame rate, then a usual frame rate at today's frame rate or camera speed. But the correct measurements made at the usual frame rate would disclose that in every film sequence the laboratories operated under the exactly same, laws of Physics, even if those laws involved Schrodinger's equation, special and general relativity!

The Theory discussed herein, involves a fictitious movie audience, consisting of astronomers viewing extremely old motion pictures, taken perhaps billions of years ago. They are imagined to be viewing a "movie" taken in the past by a variable-frame-rate movie camera! As will be discussed, we associate the frame rate of the fictitious "old movie camera of the past" with the rate or speed of time, for example frames (or seconds) per second and the time itself we associate with the length of the movie film strip that has passed though the camera. Imagine such film strip, after exposure spilling out from a broken, open camera case out on to a table. As the spent film slips along the table top from the broken camera, picturing past scenes, it would be like time moving out along the time-axis of spacetime. We don't mean to overemphasize this point, but it is critical to understanding the concept! A fast or slow speed of time is not really a new idea, as will be shown, but the idea of a variable speed of time has never before been put into the context of cosmology and a theory of our Universe. More importantly, a fundamental equation governing its operation at the beginng of Our Universe has never previously been derived. By the way, as the fictitious, ancient camera's battery approaches zero charge the movie camera approaches its end of usefulness that is the fate of our Universe!

\section{A Theory of Our Universe Is the "Big Rollout"}

Our Universe does not start out as a "Big Bang" but rather as a "Big Rollout" in space and time, spacetime from the shortest length, Planck Length, (the smallest measure of length because shorter than it, quantum effects dominate and it becomes meaningless to consider exact values of measurements) and the shortest meaningful measure of time, Planck Time (defined as the time it would take a 
photon travelling at the speed of light to cross a distance equal to the Planck Length). Please see Figure 1. It is speculated that space and time, spacetime, dimensions grow in concert very rapidly at first (actually spacetime rolling out at light speed) and, in particular, that the time dimension also grows very rapidly at first. However, as a working hypothesis the speed or rate of change of time appears to vary, according to [1]. Our Universe is similar to a malfunctioning wristwatch that is slowing down with age. As time increases, the apparent rate of its change could be erratic, that is although in general the rate of time slows approaching zero at the end of time, time's rate of change could possibly decelerate, pause or perhaps accelerate for a while since there is no apriori reason for constancy. As will be demonstrated, the speed of light will actually be constant in all time frames of reference since space and time rollout in concert.

The Rollout Theory is simpler than some portions of the theory for the Big Bang: such as "...that the nascent Universe passed through a phase of exponential expansion soon after the Big Bang, driven by a positive vacuum energy density [2]." Whereas the proposed Theory depends upon the well understood fast and slow running clocks, therefore by Occam's razor the Rollout Theory is preferable. Since the rotational rate of galaxies (leading to the concept of dark matter) and the variation of the Hubble constant (leading to the concept of dark energy) might well be dependent on the speed of time, their existence might be determined by the speed of time and might, in fact, be utilized to measure the speed of time. Note that if a clock (time) moves fast at a time in the Past, then the seconds, minutes, hours, days and years back then appear much shorter to an observer measuring them using today's time! So that processes would appear to move very rapidly in the early universe and only readily observable by detectors of high-frequency gravitational waves such as the Li-Baker [3] [4] [5].

Galaxies APPEAR to rotate faster in the past if time was moving faster back then (Figure 2). Astronomers have attributed this to a lot more mass or matter in them that holds them together so they can rotate fast and not pull apart. They call it "Dark Matter".

\section{New Theory of our Universe} My idea is very simple: our Universe starts out at "time zero" (Planck Time) and all the
coordinates of space ( $x, y, z$ or East, North, Up) are also zero (Planck Length) and then
all these dimensions, of what Einstein called "spacetime" begin to
ROLL OUT and grow and grow very fast initially and then slow down like an old clock.

"Big Bang" Alan Guth

"...that the nascent Universe passed through a phase of exponential expansion soon after the

Big Bang, driven by a positive vacuum energy density."

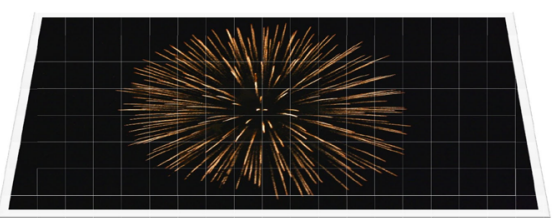

Figure 1. Theories of our Universe.
"Big Rollout" R. M L Baker, Jr. "... that our Universe Rolled Out with time moving extremely fast, and with infinitesimally small dimensions and that these spacetime coordinates are growing."

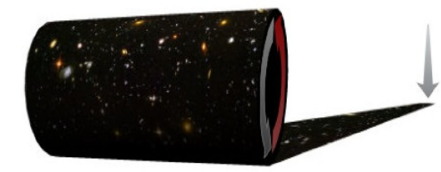




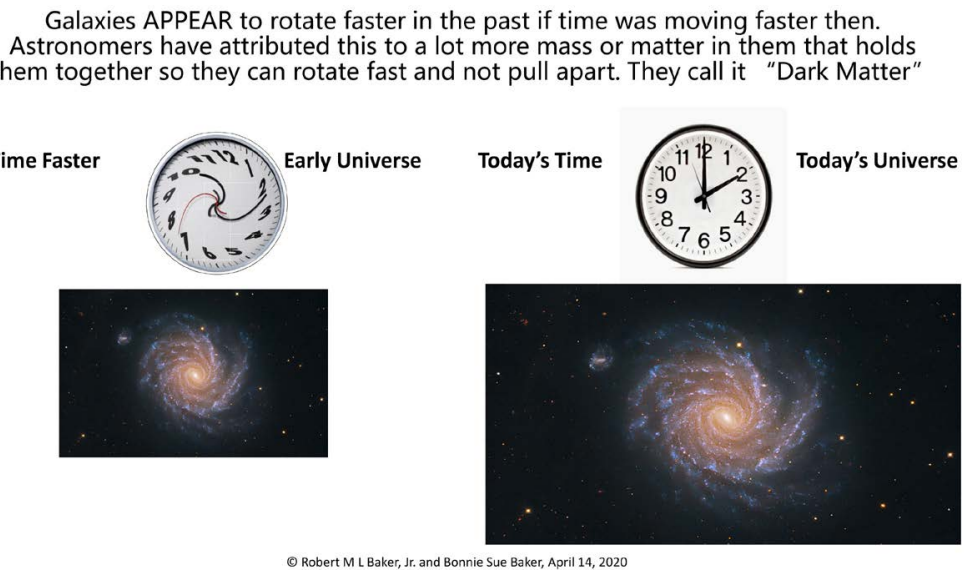

Figure 2. The speed of time slows and space grows in concert from the early universe to today's time.

\section{Dark Matter and Dark Energy}

Although dark matter and dark energy can be, in principle, predictedin the framework of extended gravity, Mars, Senovilla and Vera [6] of the University of the Basque Country, Spain, theorized that the expansion of our Universe is an "illusion" and actually is the result of the higher speed of time during the period when the light left the stellar structures in the past: “... we are fooled into thinking that the expansion of the Universe is accelerating because time itself is slowing down". So that according to J. Senovilla [7] the speed of time may be related to the "illusions" of dark matter and dark energy estimates. He suggests that the reason that we have not been able to detect dark matter may just be that it does not exist:

Those specializing in the dynamics of galaxies should analyze their motion billions of years ago as resulting from the change in the speed of time (NOT resulting from dark matter). A tool for such an analysis could be differential correction using the change in the speed of time as the parameter or local constant. One could adjust that parameter, the speed of time, by differential correction to match the observed motion of portions of a galaxy.

A notional graph of the change-of-speed-of-time variation with today's time dimension is exhibited in Figure 3. Notice the hypothetical different irregularities and slopes (tangents) especially between 1 and $10^{20}$ seconds after the "BIG ROLLOUT" or the "start" time zero or Planck Time. As theorized in Figure 3, the most dramatic manifestation of the new Theory will manifest itself for apparent times less than a picosecond after time zero.

The relationship between the change in the speed of time and the change in the space dimensions is important since they rollout in concert in order to preserve the constancy of the speed of light in any given time frame. As we look back through our telescopes and view different earlier times (time frames), the rate of time decreases and dimensions grow in inverse proportion as time moves forward. When the rate of time appears greater in the past, the dimensions appear smaller moving towards today's values. If we were able to view a stopwatch 


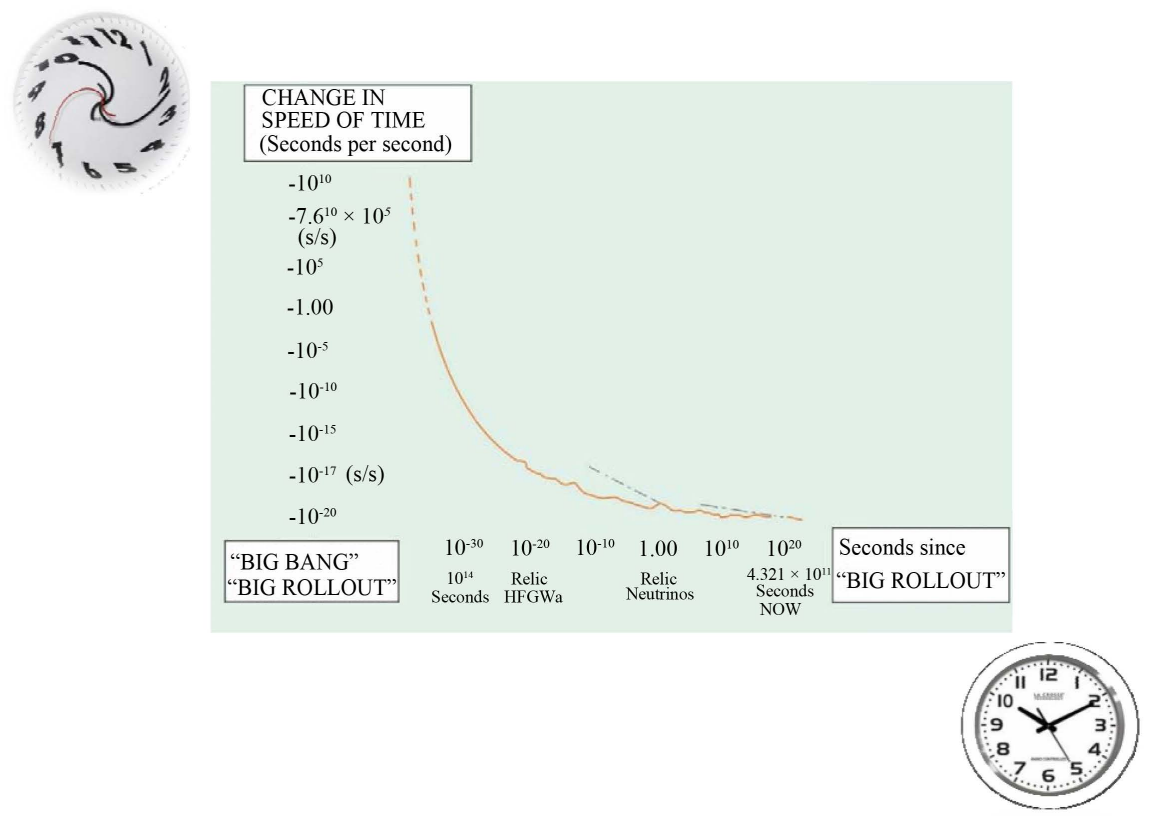

Figure 3. Notional graph of the change-of-speed-of-time variation with today's time dimension. The graph is conceptual only, it is drawn intentionally in low definition since it is NOT for numerical work. (From Figure 3 of [8]).

of a "4-minute miler" in the past, when the rate of time actually was greater, we would see its hand moving faster and we see the track is shorter as in Figure 4. If we lived back then, we would see a regular stopwatch moving at a regular rate and the ordinary one mile long track! Of course such is the case when we associate the frame rate of the hypothetical movie camera discussed in the Introduction with the rate or speed of time.

Assuming in this fictitious example that the elapsed time between observations made today (defined as time B) and the observed mile-run event (defined as time A) was 5 billion years or $5 \times 10^{9}$ years and the difference in the observed or apparent time of the event (start to finish time difference of the mile run) at time A was apparently $\left(1 / 60^{\text {th }}\right) \times(4$ minutes $)=4$ seconds or $4 \times 10^{12}$ ps and the actual time of the event at time $\mathrm{B}$ was 60 times longer or $2.4 \times 10^{14} \mathrm{ps}$, then the fictitious speed of time would be:

Speed of time (at time A) = (observed or apparent duration of the event or activity at time $A$ minus the actual duration of the event or activity at time $B$ in ps)/(elapsed time $A$ to time $B$ in years)

$$
=\left(4 \times 10^{12} \mathrm{ps}-2.4 \times 10^{14}\right) /\left(5 \times 10^{9} \text { years }\right)
$$

$=-47,200$ ps per year or $-4.7 \times 10^{-8}$ seconds per year. For comparison (page 63 of [8]), using the actual considered not fictitious and a working hypothesis Muon decay time difference (start to finish decay time difference of two consecutive Muon decays) that is $2,196,980.3 \mathrm{ps}-2,197,013.0 \mathrm{ps}=-33 \mathrm{ps}$ between 2007.0 and 2009.5 or 2.5 years elapsed time, then the speed of time was -13 ps per year in 2009.5. There is a big difference here: Muon decay time does NOT involve any space dimensions ONLY TIME! As will be discussed if somehow the 


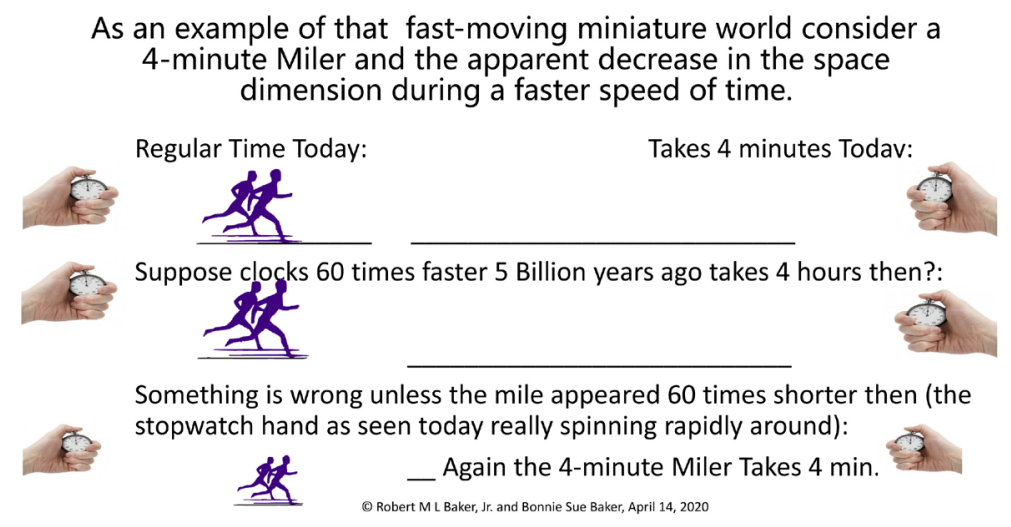

Figure 4. As a fictitious example of that fast-moving miniature world, consider a 4-minute miler and the apparent decrease in the space dimension during a time interval in which time (the stopwatch) is apparently moving faster.

events in 2007 were viewed from 2009.5, then the scene would show a slightly fast moving stopwatch measuring a slightly fast moving runner and a slightly shorter mile track. Measurements of the Muon decay time there would not involve the shrinking of any dimension and might show a slightly longer Muon decay time (more movement of the stopwatch hand during the decay process). In any event, the fictitious example of $-47,200$ ps per year ps per year for a 4 -minute miler as viewed 5 billion years ago, the -13 ps per year for Muon decay more recently determined and the notional Figure 3 seem reasonable. That is, the speed of time variation shown conceptually in Figure 3, is much faster in the past (hypothetical $-47,200$ ps per year) than at the more recent time ( -13 ps per year) as exhibited on the curve on this very hypothetical plot. The speed of time would appear to be slowing down.

In order to have the speed of light the same in all time frames of reference, as underlies the theory of special relativity, dimensions (such as the mile must be shorter if the speed of time is faster, as in Figure 5 .

At the beginning of our Universe the (change in the space dimensions) divided by the (change in the speed of time) equals (zero to the Planck Length during Planck Time)/(zero to Planck Time during Planck Time), which by the definition of Planck Time, equals the (speed of light). It is speculated that this Fundamental Equation (2) is correct at the beginning of our Universe:

$$
\begin{aligned}
& \text { (change in the space dimensions) } \\
& =(\text { speed of light }) \times(\text { change in the speed of time })
\end{aligned}
$$

Equation (2) can be rearranged as:

$$
\begin{aligned}
& \text { (change in the space dimensions)/(change in the speed of time) } \\
& =\text { (speed of light) }
\end{aligned}
$$

Applying Equation (3) to the beginning of our Universe is interpreted as meaning that initially spacetime moves out at the speed of light. Also of great importance is that this fundamental Equation (2) shows that the speed of time and the space dimensions move out in concert. As our Universe progresses, the change in a space dimension is inversely proportional to the speed of time 
The speedy photon's speed, $c=186,282.397$ miles per second and is the same in all frames of reference, which is the hypothèsis undrlying Einstein's special theory of relativity

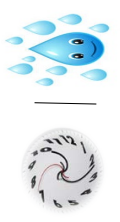

Smaller second offset by smaller "standard" mile! After time "zero" or Planck time, the speed of time slows and the space dimension grows from the infinitesimal Planck length to today's. Both changes (slow/grow) in the same proportion to insure the constancy of the photon's speed of light!

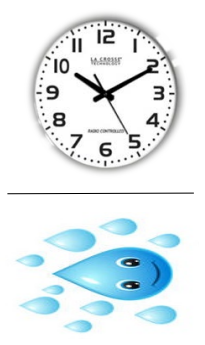

Figure 5. The speed of light is the same in all frames of reference.

change according to an extension of the fundamental Equation (2) to all times. As we look back in time the space dimensions are smaller while the speed of time is greater as shown in the left-hand side of Figure 2, that is, the little galaxy is smaller and the clock runs faster. In the fictitious example of the 4-minute miler viewed five billion years ago, having a 60 minute per minute increased speed of time. We utilize the nomenclature of Equation (1), but the event or activity is considered to be a stopwatch. At time A (five billion years ago) we view a 60 -second stopwatch (that is, the hand completes a complete circle in 60 seconds). We see it move the 60 seconds (we defined this as time A) whereas our stopwatch here and now moves only one second (we defined this as time B). So time $\mathrm{B}$ divided by time $\mathrm{A}$ is the 60 second per second speed of time indicated in Figure 4. The apparent length of the "little" mile is time A divided by time B $\left(1 / 60^{\text {th }}\right)$ of a mile as viewed today looking at the 5-billion-year-old scene! $\mathrm{A}$ 6 -foot tall runner would appear to be (time A/time B) $\times 6 \mathrm{ft}=6 / 60=1 / 10^{\text {th }}$ of a foot tall. His 2 -foot wide shoulders $2 / 60=1 / 30^{\text {th }}$ of a foot wide, his track shoes likewise shorter. So all three space dimensions are reduced proportionately as the time speed increases. Next let us consider the Muon decay time example. From [8] we find that at time A (2009.5) it required 2,196,980.3 ps for the activity of Muon decay to take place, whereas at time B (2007) it required 2,197,013 ps (remember we are looking backwards at past times). Therefore time $\mathrm{B} /$ time $\mathrm{A}=$ $1.000,015$, which shows the slightly higher speed of time in 2007 . That is, the hand of the stopwatch at time B (2007), which measures the duration of Muon decay at time $B$, had moved slightly further than the hand of stopwatch at time A (2009.5). With regard to dimensions, a 72 -inch person at time B (2007) would apparently measure $0.999985 \times 72$ inches $=71.998892$ inches as viewed in 2009.5. Of course it is totally impossible to view 2007 from 2009.5-there is no time machine. Remember, also, that at both time $\mathrm{A}$ and time $\mathrm{B}$ the person would measure exactly 72 inches tall as usual - not a billionth of an inch differently!

During observations of our Universe: distant galaxies fly away from us faster than closer galaxies, and the Hubble constant tells us how fast that is. This might be directly related to the change in the speed of time. As time increases, the rate 
of its change could be erratic as shown in Figure 3, that is although in general it slows (rate of time slows approaching zero at the end of time), its rate of change, time frame to time frame, could decelerate, pause or perhaps accelerate (speed of time increasing) for a while since there is no apriori reason for constancy. This is similar to the movie camera's variable frame rate discussed in the Introduction.

At the beginning of our Universe the "change in the space dimension" of Equations (2) and (3) does not imply that one can measure a dimension or time intervals during the initial Planck-Time "interval" because conventional physical laws no longer apply and Euclidian Geometry is completely distorted. It simply means that it represents the dimensional change from a "time zero" epoch to Planck Time at which point physical laws begin to have meaning.

We look back in time several billion years - in actuality, as pointed out in the Introduction, we do this when telescopes look at stars billions of light-years away! We may see a galaxy via 5 billion-year-old photons: It appears to be rotating faster and a little less spread out than expected. Next we look at an Olympics Games Coliseum on a duplicate Earth (we are pretending here-a thought experiment or Gedanken experiment as shown in Figure 6).

There we see a little track with little runners going around-Wow! They make 4 circuits (a mile) in a few seconds by my wristwatch! Everything a little faster and a little smaller or less spread out like the galaxy! Now I look at the weight-lifting pavilion; similar to the fictitious filming in the Introduction. There are little weight lifters also moving quickly and pushing up little bar bells. Just like the discussion in the Introduction, the laws of physics appear to be as usual to them-a fast-moving miniature World with objects getting closer together-density of our Universe increasing as we go further back in time! But densities of the barbells and their gravitational fields and obedience to Schrodinger's equation, the laws of general relativity (e.g., including proper time) are unchanged! The scenes will have a reddish hue due to the Doppler Effect.

The influence of the Doppler Effect is interesting. Please consider a classical explanation of the Effect: While sitting in a train station you hear a high-pitch sound of an approaching train whistle. As sound waves essentially pile up after leaving the approaching train, whistle and wavelengths shorten, the whistle frequency apparently increases. As the train leaves the station, the sound waves get longer and longer and their frequency decreases. However, this change in the "signal" or whistle frequency has nothing whatsoever to do with the mechanism
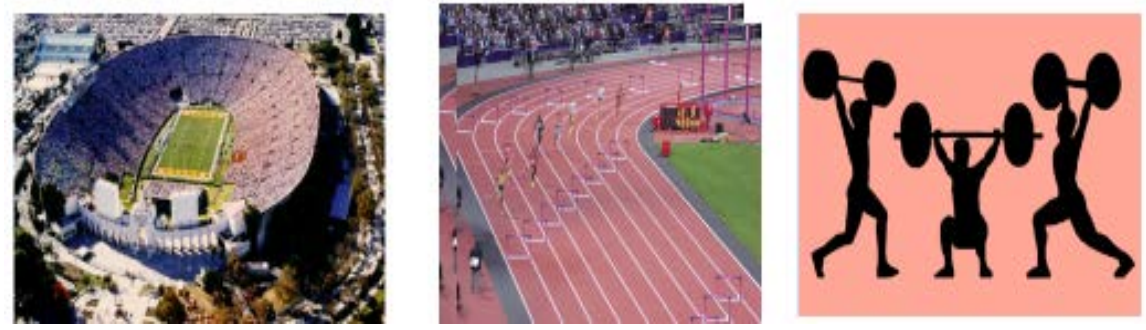

Figure 6. A fast moving miniature "World". 
of the whistle! Therefore the Doppler Effect has nothing whatsoever to do with the "mechanism" of the scene portrayed by the time frame. This same concept applies to gravitational time dilation as photons from a Scene or "frame" pass through gravitational fields on their way to your eyeball.

Figure 7 exhibits graphically the Rollout Theory with the speculated decrease in the rate or speed of time and its extremely short "years" in the early universe. Years then seem like seconds or microseconds (or even shorter, extremely close to the beginning of our Universe) as viewed today!

New mysteries. How does the speed of time vary with time itself and is there a detailed structure to that change? Does the speed of time change depend upon location and "surroundings" in our Universe (e.g., is it unique to the Earth or our Galaxy, or to change with the density of local matter, etc.) and if so what is the relationship? What is the actual theory for the change of the speed of time, that is, what is its cause? [8].

Is there a perfect clock or some kind of "absolute time"? The answer is "no." As Gyorgy Buzsaki and Rodolfo Llinas [9] in their article on "Space and time in the brain," state "... neither clocks nor brains make time per se." As was discussed in [8], Muon decay might have its own kind of "time" so one might consider Muon decay time, itself as some kind of a clock-e.g., an alarm clock. The problem is you cannot "read" it. If you ask a chef "When will the bread being baked be ready?" She might reply "I don't know exactly." I would ask then, "How do you know when it is finished and take it out of the oven?" the chef might reply "I stick a toothpick in it and if some dough no longer sticks to it, then its cooking process is over, but I do not know exactly when that will happen. I cannot read it like a clock you know!" In the context of the light cones described in Chapter 2 of [10] concerning the "arrow of time" or Entropy, there is the impossibility of distributing "polling-place clocks," which have exactly "polling-place" or absolute time, due to the special and general relativity effects as they are transported to various locations. Even if we attempt to set them by a radio signal, since we have imperfect knowledge of the speed of light (and no exact location because of Heisenberg's position uncertainty), it is impossible to accomplish the setting exactly. Time is really relative!

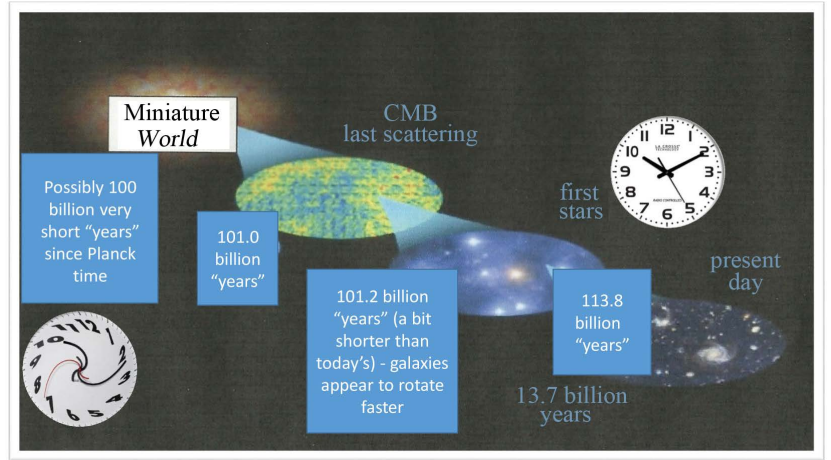

Figure 7. The growth of our Universe in local "years" that can appear today to be very short. 
A more important question is what is the reference frame for time and its rate of change? Let us suppose that you are on a boat and someone asks: "Where are we and how fast are we are going?" Also suppose you do not have GPS so you say: "I can only tell you where we are how fast we are going relative to something!" So you see a leaf nearby and say: “... well with my stop watch and yard stick I can measure that leafs' motion as we go by it and tell you my speed relative to it-of course, the leaf's actual location on our planet, and our planet's actual location in our Universe is unknown to me. Also the leaf may have its own speed relative to the water-but that is the best estimate that I have!" Therefore the only reference for time and the rate of time that we have is the clock on the wall and since, as I just suggested, there is no absolute time, so the clock on the wall "... is the best estimate that I have!" This dialog is similar to the answer you make when someone asks: "How is your wife?" The answer of course, is: "Compared to what?"

\section{Conclusion}

The fundamental equation, which when extended inversely relates the growth of the space dimensions to the slowdown of the speed of time at the beginning of Our Universe, is the key to the Theory of Our Universe or Rollout Theory. The Hubble "constant" measurements may provide a means to validate this Theory since this Hubble "constant" we believe to be essentially the separation speed of cosmic objects due to the speed of time and growth of dimensions. The Cosmic Microwave Background (CMB) occurred about 400,000 years (in length of today's apparent, observed years) after the beginning of our Universe, so that we would see separation motion of the cosmos commensurate with the speed of time then. Specifically, $6.75 \pm 0.05 \times 10^{4} \mathrm{~m} / \mathrm{s}$ per Mpc [11]. Several billion years (again today's years and seconds) after the beginning of our Universe it was measured as $7.4 \pm 1.5 \times 10^{4} \mathrm{~m} / \mathrm{s}$ per Mpc [12]. No "Dark Energy" need be assumed. The average of these two measurements is $7.07 \times 10^{4} \mathrm{~m} / \mathrm{s} \mathrm{per} \mathrm{Mpc.} \mathrm{As}$ Viktor Toth [13] points out this average "...can be expressed in terms of inverse seconds, since a megaparsec is just about 30.9 million trillion kilometers; substitute and let the length units cancel, take the inverse, and you find that the Hubble parameter is the reciprocal of about $4.4 \times 10^{17}$ seconds ( 13.8 billion, apparent, years, about the age of our Universe)... Now this is exactly the way it should be in the absence of gravitation, in a universe expanding at a constant rate. ...which means that in the past, the universe would have expanded er ..." If our interpretation of Troth's analyses is correct, then it may possibly serve to support the Rollout Theory. Analyses of Muon decay time [8] may also serve as a partial proof of the Rollout concept. In this case there is a working hypothesis that Muon decay time "...can be represented as clocks..." independent from those of our Universe. Roughly the Muon decay time's measurements, which should be a constant, decreased from 1946 to 2017 from very roughly $2,330,000$, ps to very roughly 2,110,000 ps. However, from 2007.0 to 2009.5 (the time A and time B discussed in the foregoing section) the more precise Muon 
decay time measurements exhibit a more accurate decrease in apparent Muon decay time change. If Muon decay time, like the fictitious 4-minute miler, is a "standard" marker for our Universe's time, then their time change represents the speed of time slowdown in our Universe "... of very approximately -13 ps per year' [8] from time A to time B. Also the plot of Figure 3 should be accurately developed (no longer simply an inaccurate, notional, conceptual plot) by the continued collection of Muon-decay time observations. Such data would be added to the Table on p. 62 of [8] in order to improve the accuracy and extent of the speed of time estimate. Those specializing in the dynamics of galaxies should analyze their motion billions of years ago as resulting from the change in the speed of time (NOT resulting from dark matter). A tool for such an analysis could be differential correction (Sections 1.4.2, 5.7.2, 6.7.2 of [14]) using the change in the speed of time as the parameter or local constant. One could adjust that parameter by differential correction to match the observed motion of portions of a galaxy. However, the absolutely final proof must await the development of high-frequency relic gravitational wave detectors sensitive to frequencies in the terahertz and higher gravitational-wave frequencies such as the Li-Baker [3] [4] [5]. According to Andrew W. Beckwith, Christian Corda and the author, such sensitivity would be important in the observation of the early universe at times less than a picosecond from our Universe's beginning, when this Rollout Theory predicts an extremely rapid (high frequency) activity in our new Universe! ([15] [16] [17] [18] [19] and Chapter 10 of [10]).

\section{Acknowledgements}

Andy Beckwith (my mentor in Cosmology), Alexander Fell, C. Clive Woods, Fangyu Li, Garry Stevenson, Paul Murad, Harrison Fell and Giorgio Fontana have provided support for many of my "out of the box" ideas including my Theory of Our Universe or Rollout Theory. Mounir Belgacem has given valuable assistance with the preparation of the figures and Bonnie Baker has been a beautiful inspiration and essential contributor for the entire Theory.

\section{Conflicts of Interest}

The author declares no conflicts of interest regarding the publication of this paper.

\section{References}

[1] Baker Jr., R.M.L. (2018) Analyses of the Speed of Time Based on Muon Lifetime-Decay as a Transient Time. Annual Meeting of the American Association for the Advancement of Science, Austin, 18 February 2018.

[2] Lemley, B. and Fink, L. (2002) Guth's Grand Guess. Discover Magazine, 23, No. 4, 1/8-8/8, April.

[3] Li, F. and Baker Jr., R.M.L. (2007) Detection of High-Frequency Gravitational Waves by Superconductors. International Journal of Modern Physics B, 21, 3274-3278. https://doi.org/10.1142/S0217979207044366 
[4] Baker Jr., R.M.L. (2001) Peoples Republic of China Patent Number 01814223.0 Gravitational Wave Generator and Detector. Filed July 13, 2001, Granted September 19, 2007.

[5] Woods, C.R., Baker Jr., R.M.L., Li, F., Stephenson, G.R., Davis, E.W. and Beckwith, A.W. (2011) A New Theoretical Technique for the Measurement of High-Frequency Relic Gravitational Waves. Journal of Modern Physics, 2, 498-518. https://doi.org/10.4236/jmp.2011.26060

[6] Mars, M., Senovilla, J. and Vera, R. (2008) Is the Accelerated Expansion Evidence of a Forthcoming Change of Signature on the Brane? Physical Review D, 77, Article ID: 027501. https://doi.org/10.1103/PhysRevD.77.027501

[7] Senovilla, J. (2007) Is Time Slowing Down? New Scientist, No. 2635, 5-22.

[8] Baker Jr., R.M.L. (2019) A Working Hypothesis on the Muon-Decay Time Shortening and Time. Journal of Space Science \& Technology, 25, 60-77. https://doi.org/10.15407/knit2019.03.060

[9] Buzsaki, G. and Llinas, R. (2017) Space and Time in the Brain, Science, 358, 482-485. https://doi.org/10.1126/science.aan8869

[10] Baker Jr., R.M.L. (2017) Gravitational Waves: The World of Tomorrow, a Primer, with Exercises. Infinity Publishing, Conshohocken.

[11] Alam, S., et al. (2017) BOSS Collaboration. Monthly Notices of the Royal Astronomical Society, 470, 2617-2662.

[12] Riess, A.G., et al. (2019) Large Magellanic Cloud Cepheid Standards Provide a 1\% Foundation for the Determination of the Hubble Constant and Stronger Evidence for Physics beyond $\Lambda$ CDM. Astrophysical Journal, 876, 85. https://doi.org/10.3847/1538-4357/ab1422

[13] Toth, V.T. (2020) Is It a Coincidence That If the Hubble Constant Has an Average of $70 \mathrm{~km} / \mathrm{MP}(67 \mathrm{~km}$ and $73 \mathrm{~km}$ Respectively) Then $13.8 \mathrm{~B}$ LYs of Space Will Expand Exactly at Light Speed, Same as Its Age? Quora, June 7.

[14] Baker Jr., R.M.L. (1967) Astrodynamics: Applications and Advanced Topics. Academic Press, New York.

[15] Beckwith, A.W. and Baker Jr., R.M.L. (2020) Value of High-Frequency Relic Gravitational Wave (HFRGW) Detection to Astrophysics and Fabrication and Utilization of the Li-Baker HFRGW Detector. Journal of High Energy Physics, Gravitation and Cosmology, 6, 103-122. https://doi.org/10.4236/jhepgc.2020.61010

[16] Corda, C. (2007) A Solution of Linearized Einstein Field Equations in Vacuum Used for the Detection of the Stochastic Background of Gravitational Waves. As troparticle Physics, 27, 539-549. https://doi.org/10.1016/j.astropartphys.2007.04.001

[17] Corda, C. (2009) Interferometric Detection of Gravitational Waves: The Definitive Test for General Relativity. International Journal of Modern Physics, 18, 2275-2282. https://doi.org/10.1142/S0218271809015904

[18] Corda, C. (2010) Information on the Inflation Field from the Spectrum of Relic Gravitational Waves. General Relativity and Gravitation, 42, 1323-1333. https://doi.org/10.1007/s10714-009-0895-6

[19] Corda, C. (2012) Primordial Gravity's Breath. Electronic Journal of Theoretical Physics, 9, 1-10. http://arxiv.org/abs/1110.1772 


\section{Supplementary Material}

R. M. L. Baker Jr., 2018, Invited Poster Presentation to the Annual Meeting of the American Association for the Advancement of Science, February 18.

Poster presented after session on Dark Matter Detectors

Robert M L Baker, Jr. , drrobertbaker@gravwave.com

Transportation Sciences Corporation, GravWave Division

\section{Introduction}

General Description of the problem

This presentation is based upon an observation (Discovery) by the author that the duration of Muon decay, which should be a constant, appeared to shorten from 1963 to 2017 as the years passed by. The motivation for the observation was the author's earlier conjecture that the speed of time might have been very fast in the early Universe, due to the limit on the velocity of information, and that its speed may still be slowing down from that high speed. Prior research on the speed of time had been published by Jose Senovilla, Marc Mars and Raul Vera who in 2008 speculated on time slowing related to the expansion of our Universe.
Goals of the study: Since the dawn of civilization on Earth, "time" has been an essential concern of humanity in general and Physical Science in particular.

Poincaré and Einstein both proposed a revolutionary concept that time need not move uniformly and regularly as the rate of movement of a pendulum, but that its "rate" could appear to change based upon relative speed and acceleration of clocks. But what if our clocks have been and still are slowing since they may have been moving very fast at the beginning of our Universe? That question is the subject of this presentation.

Description of methods: Unlike the intrinsic decay time of a Muon, one second is defined as the time that elapses during transition between two energy levels of the cesium 133 atom. Also such intrinsic process time of a sub system is unlike the period of a pendulum, which depends on its length and the strength of gravity. Such cesium-atom level changes and pendulum swings are the "stopwatches" of our Universe and can be utilized to measure the apparent duration of Muon decay and determine the "speed of time" (Applicution). The Proposition here is that some processes or sub systems are "marching" to their own intrinsic "time" or timeframe that is independent of the flow of "time" in our Universe. Andrew Walcott Beckwith, Report for the 27ih Solvay Conference in Physics, October 2017 Section XVII: stated: “... the issue Dr. Baker has raised is suggestive and should be thoroughly analyzed. The author finds that aside from inevitable scaling arguments, that the Muons are still a sub system, within a larger general system. i.e., the adage of Schrodinger who postulated that quantum sub systems, of a macrosystem definitely exhibit quantum mechanical time dependent behavior. Equation (51) is not quantum mechanical, but it is a sub system, and so the same rule by Schrodinger, as to sub systems exhibiting definite time dependence, may be applicable here, i.e., think in terms of time variance."

\section{Results \& Discussion}

The truth of the Proposition depends upon the measured disparity between sub system processes, which should always have the same duration in their time frame, for example Muon decay, and the duration as measured in our Universe's time frame, for example, by cesium atomic clocks. Such measurements could support or falsify the Proposition.

Of course, there may well have been overlooked systematic errors, which somehow could have been related to the particular "age" or sophistication of the measurement devices utilized or different related to the particular "age" or sophistication of the measurement devices utilized or different even though there was no real change in Muon decay time. On the other hand, such systematic errors, which have been utilized in the provisionally selected slowdown value, would have needed to errors, which have been utilized in the provisionally selected slowdown value, would have needed to
have been comprehensive of all of the five or six experimental devices, which led to the data utilized, and overlooked by all of the Muon experimenters from 1963 to 2017 and is unlikely. RESULTS:

*Over the period 2007 to 2009, the Muon lifetime change and time slowdown in our Universe near Earth has a provisional value of approximately $-\mathbf{4 1}( \pm 22)$ ps per year ( $p s=10^{-12} \mathrm{~s}$, a picosecond).

*If linear, then over 13.7 billion years $\left(1.37 \times 10^{10}\right.$ years) since the "Big Bang", clock speed would be reduced by about 0.568 seconds (small changes in the ephemerides of the planets, moons or spacecraft and galaxies appear to rotate a bit faster).

* It is speculated, however, that the speed of time decrease since the early universe could possibly be exponential starting out very fast; with time and other dimensions just "unroll out," and then gradually slowing down in the years after the Big Bang, therefore possibly affecting galaxy rotation (dark matter indicator), expansion of our Universe, etc.

(dark matter indicator), expansion of our Universe, etc. * If linear, then the clock of time would run down in $3.154 \times 10^{7} \mathrm{~s} / \mathrm{yr} / 4.1 \times 10^{-13} \mathrm{~s}=7.4 \times 10^{19} \mathrm{~s}$ and
divided by $3.154 \times 10^{7} \mathrm{~s} / \mathrm{yr}$ or $2.4 \times 10^{12}$ years or 2.4 trillion years for our Universe ("End of Time"). But again, the speed of time is speculated to be slowly decreasing (its actual variation possibly estimated bv Cepheid-variable or galactic rotation observations) so it might iușt approach zero as a References

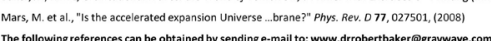

The following references can be obtained by sending e-mail to: www.drrobertbaker@growwave.com

International Solvay Institutes, section xuI (2017)

Viktor T., Toth, 2020, Quora, June 7

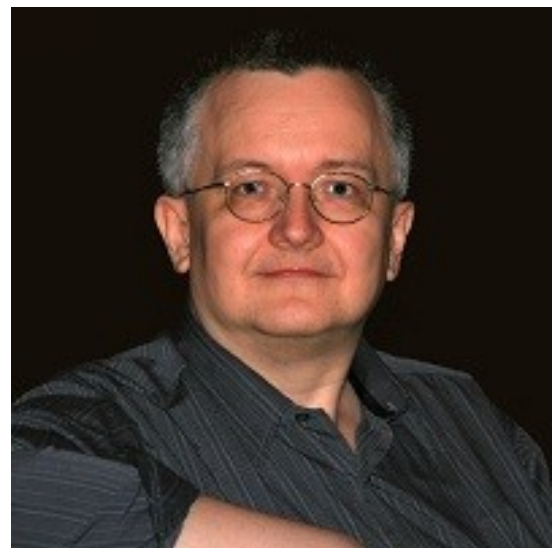

Viktor T. Toth

June 7

IT pro, part-time physicist 
Is it a coincidence that if the Hubble constant has an average of $70 \mathrm{~km} / \mathrm{MP}$ ( $67 \mathrm{~km}$ and $73 \mathrm{~km}$ respectively) then $13.8 \mathrm{~B}$ LYs of space will expand exactly at light speed, same as its age?

You stumbled upon one of the great unresolved questions in physical cosmology.

The age of the universe is 13.8 billion years, give or take, or about $4.4 \times 10^{17}$ seconds.

The Hubble parameter, which has a value of roughly $70 \mathrm{~km} / \mathrm{s} / \mathrm{Mpc}$, can be expressed in terms of inverse seconds, since a megaparsec is just about 30.9 million trillion kilometers; substitute and let the length units cancel, take the inverse, and you find that the Hubble parameter is the reciprocal of about $4.4 \times 10^{17}$ seconds.

Now this is exactly the way it should be in the absence of gravitation, in a universe expanding at a constant rate. But that is not our universe. It has gravitation. Gravitation that would normally slow down the expansion over time; which means that in the past, the universe would have expanded faster, hence it would be younger. Except, of course, that we now also have dark energy, which has been dominating the rate of expansion for the past 5 billion years or so, and its effect is to accelerate the expansion. These two effects seem to cancel each other out almost to perfection, and we end up with this strange coincidence that $t_{0}=1 / H_{0}$.

Now I should mention that in an accelerating universe, this really only happens once in that universe's lifetime. A few billion years ago, the universe would have been younger than the inverse of the Hubble parameter's value back then. A few billion years from now, it will be much older. It is only in the present epoch in the accelerating universe that the two numbers coincide.

Just how likely is it that we live precisely in this epoch? Not a couple of billion years too soon or too late for this coincidence to happen?

No one really knows the answer. It could simply be a coincidence. Sometimes.

(C)Robert M. L. Baker, Jr., June, 2020 